Mice genetically engineered to express increased levels of tumor necrosis factor developed arthritis regardless of the presence or absence of fibrin.

The authors conclude that fibrin might control the progression of inflammatory disease through a leukocyte-mediated mechanism upstream of local cytokine production. Blockade of fibrin- $\alpha_{M} \beta_{2}$ integrin interactions might be beneficial in the treatment of arthritic disease without imposing the liability of hemorrhagic risks.

Original article Flick MJ et al. (2007) Fibrin(ogen) exacerbates inflammatory joint disease through a mechanism linked to the integrin $\alpha_{M} \beta_{2}$ binding motif. J Clin Invest 117: 3224-3235

\section{Lubricin reduces friction and prevents wear and tear at the cartilage surface}

The precocious joint failure that occurs in patients with the genetic disorder camptodactyly-arthropathy-coxa vara-pericarditis (CACP) is associated with a deficiency of the glycoprotein lubricin in the synovial fluid. To determine whether lubricin's lubricant qualities prevent frictional damage to joints, Jay et al. examined the association between friction and wear in intact joints of lubricin-null mice ex vivo; the authors also examined the frictional properties of the synovial fluid of CACP patients in vitro.

The authors used a joint motion pendulum simulator to measure friction in the hind-limbs of 1-month-old and 2-month-old lubricin-null and control mice ex vivo. Microscopy studies of cartilage specimens from the knee joints of newborn, 15-day-old, 1-month-old and 2month-old mice were also conducted. The cartilage surfaces of newborn lubricin-null mice were normal, but by 2 weeks of age these surfaces appeared irregular, due to disruption of collagen fibrils, compared with those of the control mice. Friction, the cause of the wear and tear, was significantly increased in the joints of lubricin-null mice compared with those of control mice. In further experiments, purified human lubricin, visualized under an atomic force microscope, formed a meshlike network that reduced adhesion between apposing surfaces. Furthermore, synovial fluid from six patients with CACP lacked the ability of normal synovial fluid to reduce friction in an in vitro system.

The findings highlight the essential lubricating role of lubricin and suggest that the risk of joint failure could be increased by inflammation or joint trauma resulting from transient deficiencies in lubricin.

Original article Jay GD et al. (2007) Association between friction and wear in diarthrodial joints lacking lubricin. Arthritis Rheum 56: 3662-3669

\section{Is the synovio-entheseal complex involved in the pathogenesis of synovitis?}

Benjamin and McGonagle have proposed that entheses and adjacent synovium form a functional unit they term the synovio-entheseal complex (SEC). The entheses of 60 cadavers (35 male, 25 female, mean age 84 years) were analyzed to determine if evidence exists for a role of biomechanical factors of the SEC in the development of synovial inflammation associated with degenerative and inflammatory arthritis.

Of all entheses analyzed, $82 \%$ were associated with a synovial membrane and, therefore, formed an SEC. Synovial membrane was found to invade some attachment sites, similarly to pannus in inflamed joints. Histopathologic abnormalities were common in the SECs analyzed and often included fibrocartilage cell clusters, cell hypertrophy, fissuring, fibrillation and delamination, which are also found in articular cartilage in osteoarthritis. Degenerative changes were most obvious at large entheses and included necrotic areas associated with inflammatory cells. Synovial inflammatory changes existed in $85 \%$ of SECs analyzed and included inflammatory cell infiltration, lining cell hyperplasia and formation of synovial villi. Most inflammatory cells seen in the SECs were lymphocytes. Small groups of inflammatory cells (typically $\leq 10$ cells) were found in $73 \%$ of attachment sites of the entheses and were often situated near dilated venules. Since inflammatory sites were present in eight of ten entheses that did not have SECs, the authors concluded that the inflammatory infiltrates were not just a secondary consequence of synovitis.

On the basis of the observed histopathology, the authors propose an important role for SECs in 